\title{
Frauenbewegung für Staatsbürgerrechte - Das Beispiel Schweden
}

Noch vor einiger Zeit war es kaum vorstellbar, daß sich um das Konzept der Staatsbürgerschaft Debatten entzündeten, die von Geschlechtskategorien ausgingen. Das Konzept Staatsbürgerschaft (»citizenship «)* war an Universalität gebunden, an eine Person, die ein Bündel von Rechten und Pflichten verkörperte. Innerhalb des universellen Rahmens zeichnete sich allerdings die Figur eines männlichen Bürgers ab: Es war der unabhängige »pater familias« im liberalen Staat, der männliche Ernährer und seine Familie nach Marshalls (1950) Gefüge sozialer Staatsbürgerrechte oder der rechtmäßige Vertreter des unabhängigen Haushalts wie bei Rawls (1971). ${ }^{1}$ Die jüngere feministische Forschung hat nun unter einer geschlechtlichen Perspektive versucht, die begrifflichen Grenzen von Staatsbürgerrechten neu zu definieren und zu erweitern, indem sie Schlüsselbegriffe in den vorherrschenden Theorien dekodierte: Abhängigkeit/Unabhängigkeit, privat/öffentlich, Bedürfnisse/Rechte (Fraser und Gordon 1994; Knijn 1994; Saraceno 1994).

Das feministische Projekt, Staatsbürgerschaft zu vergeschlechtlichen, ist in mancher Hinsicht Teil eines umfangreichen Programms zur begrifflichen Erweiterung des Bürgerrechtsparadigmas. Gleichzeitig ist Staatsbürgerschaft zu einem in weiten Bereichen umstrittenen Konzept geworden. In der europäischen Gemeinschaft und Politik läßt sich der Zusammenbruch des Sowjetregimes nicht ignorieren, der in den postkommunistischen Gesellschaften zu einer Neubestimmung von Staatsbürgerschaft führte, die politischen Identitätsbedürfnissen in bezug auf Nation und Ethnie entsprach und Kriege auslöste. Nicht nur der Zerfall in Nationalstaaten, sondern auch die Frage von Bürgerrechten außerhalb nationaler Grenzen steht heute auf der Tagesordnung: Eurobürgerschaft, Weltbürgerschaft, oder auch Bürgerrechte innerhalb gedachter Gemeinschaften unter Homosexuellen, Pazifisten, Umweltschützern, Feministinnen (De Swann 1995; Turner 1992; Jones 1994). Unter Sozialwissenschaftlern wurden in Zeiten der Beschneidung des Wohlfahrtsstaats und des Rollbacks Staatsbürgerrechte zu einem strategischen Ansatzpunkt für eine Rückbesinnung auf die theoretischen Grundlagen von

* Vgl. zum Problem der Übersetzung dieses Begriffs ins Deutsche den Beitrag von Ute Gerhard in diesem Heft. 
Rechten, sozialer Zugehörigkeit und politischer Partizipation (Lister 1996; Turner 1992).

Der neue Diskurs über Staatsbürgerschaft ist dynamischer geworden und geht über die konventionellen Bestimmungen als Status (formale Rechte) aber auch als Identität hinaus, indem er die Dimension partizipatorischer Bürgerschaft in der Tradition der griechischen Demokratie und Rousseaus wiedereinführt. Dies sind Traditionen, aus denen Frauen bewußt ausgeklammert blieben. Es ließe sich aber auch behaupten, daß der Rahmen partizipatorischer Bürgerschaft eine theoretische Ausgangsbasis liefert, um die Rolle von Frauen als soziale Akteurinnen bei der Herausbildung von Staatsbürgerrechten genauer zu bestimmen.

In einer Reihe von Büchern und Artikeln zur neueren Geschlechterforschung ist der Mangel einer Theorie weiblicher Handlungsfähigkeit (»agency «) und der entsprechenden bürgerrechtlichen Parameter immer wieder deutlich gemacht worden (Hobson und Lindholm 1996; Dietz 1992; Lister 1996; Siim 1994; Skopcol 1992). Einige der feministischen Wissenschaftlerinnen, die sich mit Staatsbürgerrechten in der Geschlechterperspektive auseinandersetzen und die Relevanz partizipatorischer Rechte erkannt haben, beriefen sich auf Hannah Arendt (vgl. Honig (Ed.) 1995). Obwohl Geschlecht als historische Kategorie oder Frauen als besondre Staatsbürgerinnen in Arendts Analyse überhaupt nicht vorkommen, haben diese Feministinnen in deren Schriften über Staatsbürgerschaft und Demokratie eine vitale Vorstellung von Staatsbürgerschaft und vom politischen Raum gefunden. So wie Arendt umstrittene Momente oder »verlorene Geschichten " in der Entstehung demokratischer Gesellschaften wieder entdeckt hat, so wollen sie eine Frauenpolitik und Momente geltend machen, in der Frauen als gesellschaftlich Handelnde sichtbar waren.

Es ist verständlich, daß Arendts radikaler Begriff des Politischen für feministische Wissenschaftlerinnen verführerisch ist, denn er überschreitet das eingegrenzte Feld formaler Politik und nationaler politischer Parteien, in dem Frauen nur selten Schlüsselpositionen einnehmen. Aber bei dem Versuch, das Handeln von Frauen und die Konstruktion von Staatsbürgerrechten zu theoretisieren, bringt Arendt uns nicht weiter. In ihrer Weigerung gesellschaftliche oder »private « Räume als politische Bereiche gelten zu lassen, blendet sie weite Teile des Lebens von Frauen aus (Landes 1995). Und sie gibt wenig Ansatzpunkte für ein Problem, das in der feministischen Literatur zentral geworden ist, daß nämlich Partizipation und Zugang zur Politik in hohem Maße von sozialen Rechten abhängen.

Wenden wir uns T. G. Marshalls Analyse sozialer Rechte zu. Wie bei Arendt, sind auch in seiner Community die Bürger entkörperte Individuen: sie gebären keine Kinder, sie sorgen nicht für Familienangehörige und erfahren auch keinen geschlechtsbedingten Ausschluß von der vollen Teilhabe am gesellschaftlichen und politischen Leben. Marshall erkannte die Verbindungen zwischen politischen und sozialen Rechten. In seiner Sequenz bürgerlicher, politischer und sozialer Rechte folgen die sozialen auf die politischen Rechte und sind ein Resultat von Machtausübung. Macht auszuüben, be- 
deutete für ihn jedoch, zu wählen. Und so gab es bei ihm nur wenig Spielraum für die Machtausübung von unten, etwa durch Graswurzelpolitik vor der Erlangung des Wahlrechts.

Frauenparteien sind mit Ausnahme von Island noch nie eine nennenswerte politische Kraft bei Wahlen gewesen. In der Vergangenheit sind Frauen von den Organisationen der Arbeiterbewegung marginalisiert worden, und auch heute verfügen sie selten über Macht in den Gewerkschaften. Um $z u$ verstehen, wie Frauenkollektive Staatsbürgerrechte ausgeweitet haben, müssen also andere Modelle der Machtausübung entwickelt werden. Dazu gehört eine neue Theoretisierung staatsbürgerlicher Praxis (Jones 1994; Phillips 1991; Siim 1994), die Nachbarschaften, Arbeitsplätze, politische Institutionen und soziale Bewegungen miteinbezieht. Die Phase kollektiver Identitätsbildung, die für eine Theorie politischer Handlungsfähigkeit von Frauen im Rahmen des Bürgerrechtskonzepts überaus wichtig ist, nenne ich composing of constituency. Dieses Konzept umfaßt sowohl den Prozeß, in dem sich unter den einzelnen Mitgliedern einer Gruppe ein gemeinsames Bewußtsein und geteilte Meinungen entwickeln, die Kennzeichnung von Problemlagen und Zielen innerhalb einer sozialen Bewegung, als auch die Repräsentation der Gruppe in öffentlichen Arenen. Mit der Prägung des Begriffs composing constituency möchte ich die Bedeutung sozialer Akteure und dynamischer Identitätsbildungsprozesse hervorheben sowie die historischen Zufälle und unerwarteten politischen Chancen betonen. Ich gebrauche den Begriff nicht in einem engeren Sinn von Wählerschaft (voting constituencies), sondern im erweiterten Sinne: als Repräsentation gesellschaftlicher Gruppen in verschiedenen Arenen des Diskurses, in Texten, die für ein größeres Publikum bestimmt sind, in innerparteilichen und überparteilichen politischen Auseinandersetzungen.

Um staatsbürgerliche Praxis tatsächlich zu begreifen, bedarf es eines Wissens über die Kontexte der Herstellung geschlechtlicher Identitäten, d.h. über die Phasen, in denen sie allmählich Gestalt annehmen. Wird mit der Phase vor der Formgebung begonnen, in der politische Handlungsfähigkeit sich herausbildet, so kann das Dilemma zwischen universellem und partikularem Staatsbürgerstatus umgangen werden, das gelegentlich als Dilemma von Gleichheit und Differenz auftritt. Ein prozessuales Modell der Identitätsbildung (the composing of constituency) gibt einen Rahmen ab für die Erkenntnis von 1. verschiedenen Identitäten in der Kategorie Frauen und 2. für die Konvergenz von Überzeugungen und Visionen, die in der staatsbürgerlichen Praxis zum Ausdruck kommen.

In diesem Aufsatz möchte ich die Bürgerrechtspraxis von Frauen darstellen, indem ich Konturen ihrer Identitätsbildung und die Artikulation ihrer Ansprüche in einer besonderen politischen Landschaft nachzeichne: Schweden in den 1930er Jahren. ${ }^{2}$ In diesem Jahrzehnt bildet sich mit dem Beginn der langen Regierungsperiode der Sozialdemokratie der schwedische Wohlfahrtsstaat heraus. Dies ist auch eine Blütezeit politischer Frauenorganisationen. Es geht mir nicht darum, den Fall Schweden als typisch oder paradigmatisch hinzustellen, und auch nicht darum, ihn zur Norm eines weiblichen 
Staatsbürgerstatus zu erheben. Meine Absicht besteht vielmehr darin, ein Modell für dynamische Prozesse vorzustellen, an dem sich zeigt, wie und wann das Handeln von Frauen die Konturen des Bürgerstatus' im Wohlfahrtsstaat prägt.

\section{Politische Handlungsfähigkeit entwickeln}

Politische Mobilisierung ist für die Aktivistinnen unter Frauen in der Vergangenheit aus verschiedenen Gründen sehr schwierig gewesen und ist es nach wie vor. Erstens wurden Frauen von den üblichen Wegen in die Organisation und den Kanälen innerhalb derselben direkt oder indirekt ausgeschlossen. Selbst wenn Gewerkschaften und politische Parteien, die traditionellen Organisationsformen der Mobilisierung und der Machtbildung, den Frauen die Mitgliedschaft nicht verweigerten (was in weiten Teilen der britischen und amerikanischen Gewerkschaftsbewegung der Fall war), so konnten sie dort doch nur eine marginale Rolle spielen. Außerdem hat die soziale Isolierung von Frauen, die im Haus oder auf einem Hof arbeiteten, die Entstehung von Netzwerken und organisatorischen Verbindungen sehr behindert. Für Frauen, die ökonomisch vom Ehemann abhängig sind, ist es darüber hinaus schwierig, Zeit für politische Aktivitäten auszuhandeln. Das ist ein Beispiel dafür, daß politische/partizipatorische Rechte auf sozialen Rechten beruhen. Schließlich geben die unterschiedlichen Erfahrungen und verschiedenen sozialen Hintergründe oft Anlaß zum Streit, und sie stellen ein Hindernis dar für die Herausbildung stabiler Organisationen, die »Fraueninteressen « repräsentieren könnten.

Wie viele europäische Frauenbewegungen in den ersten Jahrzehnten des zwanzigsten Jahrhunderts, so waren auch die schwedischen Frauengruppen durch Klasse, politische Loyalität und ideologische Uneinigkeit in bezug auf Schutz und Rechte gespalten. Die Geschichte der schwedischen Frauengruppen ist eine Geschichte verschiedener Organisationen mit unvereinbaren Zielen. Während des ganzen neunzehnten Jahrhunderts waren sie durch soziale Schicht, städtische und ländliche Umgebung und politische Zuordnung voneinander getrennt (Lindholm 1991). Die Frederika-Bremer-Gesellschaft, die sich selbst mit Stolz als rein feministische Organisation darstellte, wurde 1885 von städtischen Oberschichtfrauen gegründet, die Erziehungsreformen und pädagogische Berufe anstrebten (Evans 1977). Land- und Mittelschichtfrauen waren eher bereit, sich den Tolfterna (Dutzend) genannten Selbsterfahrungsgruppen anzuschließen, die von Ellen Key ins Leben gerufen wurden, der Protagonistin eines maternalistischen Zukunftsentwurfs. 1892 gründeten sozialdemokratische Frauen eine eigene Organisation, um die Frauen der Arbeiterklasse in ihrem politischen Engagement zu unterstützen. Der Kampf für das Wahlrecht brachte verschiedene Gruppen schließlich zusammen, aber die schwedische Stimmrechtsbewegung war nicht von so langer Dauer wie die angloamerikanische und brachte auch keine vergleichbare nationale Massenbewegung hervor. 
Nach der Erlangung des Wahlrechts wurde die Klassen- und Parteienspaltung reaktiviert und vertiefte sich. Für die schwedischen Frauengruppen führten ideologische Unterschiede wegen der festsitzenden Klassenantagonismen zu tieferen Brüchen und Einschnitten als anderswo. Die bürgerlichen Frauen fürchteten die Rede von Revolution und Klassenkampf, während die Frauen aus der Arbeiterklasse ihr Mißtrauen gegenüber Mittelschichtorganisationen äußerten. Als zum Beispiel 1919 die nationale schwedische Hausfrauenvereinigung gegründet wurde, waren die Mitglieder der sozialdemokratischen Frauenvereinigung aufgrund der Mittelschichtorientierung dieser Organisation sehr mißtrauisch und fragten: »Bedeutet das, daß sie zum Sprachrohr aller Frauen geworden sind? Wir müssen ihre Aktivitäten sehr genau im Auge behalten« (Åkermann 1983,135).

Gegen Mitte der dreißiger Jahre hatten die Klassenspannungen zwischen den Frauengruppen aufgrund zahlreicher Kooperationsbeziehungen einigermaßen nachgelassen. Um sich als loyale Anhängerinnen von Gleichheitsstreben und Demokratie hinzustellen, hatten Mittelschichtfrauen auch Frauen aus der Arbeiterklasse in ihre Organisationen aufgenommen (Åkermann 1983,195). Eine feministische Autorin schrieb 1939 in der sozialdemokratischen Frauenzeitung Morgonbris (Morgenbrise), daß es eine weitverzweigte Bewegung von Frauengruppen gäbe:

"Ich habe die Erfahrung gemacht, daß sich in der Frauenbewegung eine Veränderung vollzogen hat. Von einem rein bürgerlichen Phänomen, das gegen den großen Widerstand von Frauen der Arbeiterklasse unter der Führung von Mittelschichtfrauen stand, ist sie zu einer Bewegung geworden, die alle angeht" (Morgonbris 1939/12, 5).

$\mathrm{Zu}$ dieser Zeit hatten die Anführerinnen der schwedischen Frauenbewegung zwei Hindernisse zu überwinden: Sie fanden eine in Fraktionen zerfallene Bewegung vor, für die Werte und Ziele gefunden werden mußten, die unabhängig von verschiedenen Klassen, Parteien und gesellschaftlichen Rollen, von allen Gruppen geteilt werden konnten. Außerdem standen sie vor der Aufgabe, zu bestimmen, welche Rolle Frauen in der Öffentlichkeit spielen sollten und worin ihre Partizipation als politische Akteurinnen bestehen sollte.

Politische Willensbildung und die Herstellung von Handlungsfähigkeit (composing a constituency) erfordert nicht nur, sich auf einen Kern von Problemen oder Mißständen festzulegen, sondern es muß auch ein gemeinsamer Rahmen gefunden werden, der bei den Angehörigen eines Kollektivs Resonanz findet. To compose bedeutet buchstäblich (nach seinen lateinischen, altenglischen und französischen Wurzeln), an einem Ort zusammenzukommen. Durch Analyse der kognitiven Prozesse der Meinungsbildung (cognitive framing) innerhalb sozialer Bewegungen können wir die Spuren der kollektiven Identitätsbildung nachzeichnen und deren Einfluß auf die Auswahl von das Zustandekommen von Zielen erkennen. 


\section{Kognitive Rahmung und Kollektividentitäten}

In Theorien sozialer Bewegung wird als kognitive Rahmung jener Proze $B$ bezeichnet, durch den sich die Überzeugungen oder die Weltanschauung der einzelnen Teilnehmer mit dem ideologischen Rahmen der Gesamtorganisation der Bewegung verbinden (Snow/Benford 1986, 1992). Der kognitive Rahmen einer Bewegung ist die Basis für die Herausbildung von Gruppenloyalitäten. Er gibt vor, in welcher Weise soziale Gruppen ihre Forderungen artikulieren, er enthält ihr Potential an Bündnisfähigkeit, und schließlich bestimmt er auch, welche Möglichkeiten die Gruppen finden, um neue Gesetze und Politiken einzuführen, die die Grenzen des Staatsbürgerstatus erweitern.

In der Forschung zu den Frauenbewegungen der ersten Jahrzehnte des Jahrhunderts ist häufig der Begriff »Maternalismus « aufgenommen worden, um damit den Aktivismus jener Frauen zu kennzeichnen, die Fraueninteressen als Interessen von Müttern verstanden. Tatsächlich war Maternalismus im Rahmen jener Bewegungen ein entscheidendes Moment. Ich möchte aber behaupten, da $\beta$ dabei nicht nur verschiedene Subtexte berücksichtigt werden müssen, sondern daß die Frauenbewegungen (Koven/Michel 1993; Ladd-Taylor 1994) in den skandinavischen Ländern tatsächlich für sich ein anderes System konstruierten. ${ }^{3}$ Der kognitive Rahmen für die Frauenbewegungen in Schweden und anderen skandinavischen Ländern war vorrangig an Staatsbürgerrechten und partizipatorischer Demokratie orientiert. Schwedische Feministinnen trugen ihre Vorstellungen vielfältiger Identitäten von Frauen zusammen, der von Müttern, Staatsbürgerinnen und Arbeiterinnen.

Es gab eine maternalistische Tradition im schwedischen Feminismus. Deren Hauptprotagonistin war Ellen Key, sie machte geltend, daß die Macht einer Frau sich aus ihrer Mutterrolle und aus ihrem weiblichen Bewußtsein herleite. Diese Einstellung, die eine essentialistische Auffassung der Geschlechterdifferenz einschloß, verlor allerdings in den dreißiger Jahren an Boden. ${ }^{4}$ Seit den Zwanzigern und während der Dreißiger bildeten schwedische Feministinnen ihre politische Identität im Zusammenhang mit staatsbürgerlichen Konzepten und demokratischer Teilhabe an der Regierung heraus. Sie behaupteten tatsächlich, es gäbe keine besonderen Frauenfragen: alles beträfe auch die Frauen und was Frauen dächten, ginge jeden etwas an (Eskillsson 1991).

Die feministischen Gruppen in Schweden trafen sich untereinander, es gab Auseinandersetzungen zwischen den verschiedenen Organisationen. Sie definierten schließlich ihre Ziele und legitimierten ihren Aktivismus im Rahmen von Bürgerrechten und partizipatorischer Demokratie. Angesichts des schwachen politischen Engagements von Frauen - viele machten noch nicht einmal von ihrem Wahlrecht Gebrauch - starteten die Anführerinnen von Frauenorganisationen eine Kampagne zur stärkeren Beteiligung von Frauen in der Politik. Politik wurde in einem sehr weiten zivilgesellschaftlichen Sinne verstanden, es galt, sich als Bürgerin zu informieren und an der 
Entwicklung neuer politischer Strategien zu beteiligen. Die Frauengruppen bereiteten ihre Mitglieder auf die politische Betätigung durch Lektüren und Seminare vor. In den Schulungen ging es sowohl um Gegenstände aus der politischen Theorie als auch um praktische Fertigkeiten, wie etwa Redetechniken. Eine Feministin erklärte: "Die Frauenbewegung braucht ihre Arbeitsstätten, einen Ort, an dem ihre Mitglieder für die Wahrnehmung ihrer gesellschaftlichen Pflichten vorbereitet werden « (Spoof 1933,4). Eine sehr einflußreiche "Ausbildungsstätte " war die Bürgerschule, die von Feministinnen der Fogelstad-Gruppe gegründet worden war. Teilnehmerinnen der Bürgerschule waren in den dreißiger Jahren sehr aktiv und ihre Ideale gingen in die damalige Frauenbewegung ein.

Kerstin Hesselgren, die erste Fabrikinspektorin und das erste weibliche Parlamentsmitglied, war ein Mitglied der Fogelstad-Gruppe gewesen. Sie bestimmte den Staatsbürgerstatus von Frauen als Partizipation. Sie vertrat die Auffassung, daß die Stimmen der Frauen zur Aufrechterhaltung einer demokratischen Gesellschaft unabdingbar seien, implizit gehörten zu diesem Rahmen von Staatsbürgerschaft auch Rechte und Pflichten: "Unsere zukünftige Demokratie muß von einer aufgeklärten weiblichen Wählerschaft unterstützt werden. Die ungebildete, passive Bürgerin ist eine direkte Bedrohung für unsere Demokratie « (Thorell 1969, 2).

Bündnisse über Klassen- und Parteiengrenzen hinweg führten zu neuen, innovativen staatsbürgerlichen Praktiken. 1936 unterzeichneten fünfundzwanzig Frauenorganisationen einen offenen Brief, der Frauen zu stärkerer politischer Repräsentation aufrief. Dieser gemeinsame Brief mit der Überschrift »Ein Aufruf an die schwedischen Frauen « wurde sowohl von der Organisation der Postarbeiterinnen unterzeichnet als auch von der der schwedischen christlichen jungen Frauen (Arbetets Kvinnor 1932, 21f.), um nur zwei Beispiele aus dem breiten Spektrum zu nennen. Er wurde in gewerkschaftlichen und in bürgerlichen Frauenzeitschriften veröffentlicht. Er forderte die Frauen auf, durch den Beitritt zu einer Partei und den aktiven Einsatz für Politikerinnen zur Förderung von Frauen beizutragen. Interessant ist, daß der Brief sich auf keine bestimmte Parteilinie festlegte, sondern Frauen aller Schichten lediglich dazu aufrief, sich überhaupt einer Partei anzuschließen, gleich welcher.

Als allgemeine Ziele waren schon früher formuliert worden: 1. Die schwedischen Frauen sollten zu würdigen Mitgliedern der Gesellschaft werden. 2. Eine Offensive zur politischen Gleichstellung von Männern und Frauen sowie 3. zur ökonomischen Gleichstellung. 4. Die neuerworbenen Frauenrechte sollten geschützt werden. 5. Die Solidarität unter Frauen müsse gestärkt werden (Svenska Kvinnors Medborgarsförbund 1931, 23).

Oberflächlich betrachtet sind diese Zielsetzungen nicht revolutionär oder provokativ. Aber zu jener Zeit mögen einige der Zielsetzungen auf die dominanten politischen Parteien in Schweden etwas entmutigend gewirkt haben. Die Vorstellung von Solidarität unter Frauen lief auf Loyalitäten jenseits von Klasse und Partei hinaus. Wir dürfen annehmen, daß sozialdemokratische Männer ein ziemliches Unbehagen entwickelten angesichts der ge- 
sellschaftlichen und ökonomischen Gleichberechtigungsbestrebungen. Es waren ja nicht einfach »Rotstrümpfe«, sondern Arbeiterinnen, die über Benachteiligung und Ungleichheit zwischen Männern und Frauen sprachen, statt den Klassengegensatz im Munde zu führen, der die politische Rhetorik der Sozialdemokraten damals noch bestimmte.

In den dreißiger Jahren bekamen die Frauenorganisationen erstaunlichen Zulauf. In der sozialdemokratischen Frauenbewegung stieg die Zahl der Mitglieder von 7302 im Jahre 1930 auf 26882 im Jahre 1940. Die Adressenliste der nationalen Hausfrauenvereinigung wurde in diesem Jahrzehnt immer größer, so daß 1940 mehr als 23550 Frauen die Mitteilungsblätter bekamen, gegenüber 10000 im Jahr 1930 (Lindholm 1990, 88, 99). Das ist bemerkenswert, denn ein Zahlenvergleich ergibt, daß jetzt fast doppelt so viele Frauen organisiert waren wie bei der Wahlrechtskampagne (Lösman 1987, 199). 1937 umfaßte die Frederika Bremer Gesellschaft 36 Ortsgruppen mit über 6000 Mitgliedern und die Organisation schwedischer Geschäftsfrauen hatte Ende der Dreißiger stolze 4000 Mitglieder (Lindholm 1990, 108).

Es gab außerdem Netzwerke und einen lebendigen Austausch zwischen Feministinnen in verschiedenen Organisationen, die Frauen unterschiedlicher gesellschaftlicher Herkunft und politischer Orientierung mit diversen Reformprogrammen repräsentierten. Sie hielten Reden bei den jeweiligen Treffen der anderen, publizierten in den jeweiligen Zeitschriften und kooperierten in einer Reihe von Fragen. Die Koalition war erfolgreich, denn sie führte sowohl zu Kompromissen als auch zu wechselseitiger Unterstützung bei bestimmten inhaltlichen Fragen der Einzelorganisationen. So arbeiteten z.B. die sozialdemokratischen Frauen mit der Akademikerinnen- und der Hausfrauenorganisation zusammen, um das praktische Wissen von Hausarbeit zu vergrößern (Flood 1960, 202). Sie konnten Rationalisierungsvorschläge machen zur besseren Vereinbarung von Ehe, Mutterschaft und Arbeit (Lindholm 1990, 121). Gleichzeitig unterstützte die Hausfrauenorganisation in ihrem Mitteilungsblatt den gesetzlichen Schutz weiblicher Erwerbsarbeit bei Ehefrauen und Anträge zur Entkriminalisierung der Abtreibung (Wohlin 1935).

\section{Beschwerden und Forderungen}

Der bürgerrechtliche Rahmen bestärkte die schwedischen Frauengruppen darin, einheitlich auf die drohende Einschränkung des Erwerbsrechts von Frauen zu antworten, die während der Wirtschaftskrise in vielen Industrienationen am Horizont auftauchte. In Schweden war das Recht auf Arbeit ein Kernprinzip der Sozialdemokratie. Frauen dieses Recht zu verweigern, wäre einem Angriff auf ihre Anerkennung als Staatsbürgerinnen gleichgekommen.

Weltweit waren die Frauenbewegungen in den dreißiger Jahren in der Defensive. In vielen Ländern wurden die Frauen verantwortlich gemacht für 
das hohe Arbeitslosenniveau und sie wurden beschuldigt, die Löhne in eine Abwärtsspirale zu bringen. ${ }^{5}$ Die politische Auseinandersetzung über das Recht verheirateter Frauen auf Erwerbsarbeit fing in Schweden vor dem Hintergrund hoher Arbeitslosigkeit an. Die Zahl der Arbeitslosen war zwischen 1931 und 1932 von 32000 auf 161000 angestiegen und erreichte 1933 mit 186000 registrierten Arbeitslosen ihren Höhepunkt (Tingsten 1973, 285). Der Widerstand gegen die Erwerbstätigkeit verheirateter Frauen war so gro $B$, daß alle Parteien, rechte wie linke, Restriktionen forderten. Im schwedischen Parlament wurden 1934 mindestens neun Anträge zur Einschränkung der Erwerbsarbeit von Ehefrauen gestellt.

Einige schwedische Frauen hatten ein handfestes Interesse am Fortbestehen des Rechts auf Erwerb (10\% aller weiblichen Erwerbstätigen waren Ehefrauen), sehr viel mehr begriffen allerdings, welche symbolische Bedeutung der Verteidigung dieses grundlegenden Bürgerrechts zukam. So wurden in Schweden als direkte Antwort auf die Gefährdung dieses Rechts Zweigstellen der Open Door International und der International Federation of Business and Professional Women eingerichtet (Wikander 1992, 42). Das gesamte Spektrum der Frauenorganisationen einschließlich der Hausfrauenorganisation verteidigte das Recht auf Arbeit mit der Begründung, daß es ein Bürgerrecht sei. Und obwohl sie es lieber gesehen hätten, daß die Mütter zu Hause blieben, wandten sich die Sprecherinnen der Hausfrauenorganisation gegen einen Vorschlag, Ehefrauen die Frühverrentung anzubieten und sie mit einer Abfindungssumme zu entschädigen (SOU 1938). Innerhalb des Bürgerrechtsrahmens wurde das Recht auf eine einträgliche Beschäftigung als das Recht behandelt, "einen Beitrag für die Gesellschaft zu leisten « (Koch 1935, 2f.). Es war eine Voraussetzung, in verschiedenen Lebensbereichen als Arbeiterin, Mutter und Bürgerin aktiv teilzunehmen.

Den schwedischen Frauenorganisationen gelang es in dieser Zeit nicht nur, den Angriff auf das Recht auf Erwerbstätigkeit von Ehefrauen ${ }^{6}$ abzuwehren, sondern sie konnten sogar gesetzliche Regelungen durchsetzen, die auf eine Ausweitung des Frauenarbeitsrechts hinausliefen. Und das zu Zeiten der Wirtschaftskrise, als Frauen in ganz Europa und Nordamerika dieses Recht verloren. 1938 wurde ein Gesetz verabschiedet, das untersagte, Frauen zu entlassen, die verheiratet, schwanger oder alleinstehende Mütter waren.

Wenn wir uns im Vergleich dazu mit der zur selben Zeit stattfindenden Kampagne gegen die Diskriminierung von Ehefrauen in der Arbeitsgesetzgebung in den USA befassen, so wird deutlich, daß die vorherige kognitive Festlegung auf weibliche Gemeinsamkeiten, die sich auf die Rolle der Kinder gebärenden und umsorgenden Mütter bezog, die feministische Kampagne für den Schutz des Rechts der Frauen auf Erwerbstätigkeit einigermaßen schwächte. Die Spaltungen, die durch konkurrierende kognitive Rahmen in der US-amerikanischen Frauenbewegung entstanden, zwischen denjenigen, die sich affirmativ auf maternalistische weibliche Identitäten bezogen und denjenigen, die auf der Gleichberechtigung von Frauen beharrten, sind nicht zu unterschätzen. Als die amerikanische Frauenpartei 1920 
das Equal Rights Amendment als Hauptstrategie zur Abschaffung der Ungleichheit zwischen Männern und Frauen einbrachte, teilte sich die amerikanische Frauenbewegung in verschiedene Fraktionen - hauptsächlich unter den Mittelschichtfeministinnen - auf. Die einen glaubten, das Equal Rights Amendment gefährde Programme zur Unterstützung von Müttern und besonders von erwerbstätigen Müttern, wie etwa die Arbeitsschutzgesetzgebung. Die anderen wiederum betrachteten solche geschlechtsspezifischen Programme und Gesetze als Hindernisse für die gleichberechtigte Teilnahme von Frauen am Arbeitsmarkt (Harris 1988). Obwohl die Mehrheit der amerikanischen Feministinnen gegen die Ausnahmegesetze für verheiratete Frauen war, die in den Dreißigern durchgesetzt wurden, unterminierte die sichtbare Spaltung zwischen den Frauengruppen doch die Position und die Ansprüche derjenigen Feministinnen und Organisationen, die öffentlich als Vertreterinnen von Fraueninteressen auftraten (Hobson 1993). Während der zwanziger Jahre wollten die amerikanischen Feministinnen, die einen Mutterschaftsdiskurs führten, die Position von Frauen durch eine besondere Gesetzgebung für Mütter stärken. Es ging um Witwenpensionen, die gesundheitliche Situation von Müttern, besondere Schutzbestimmungen für Mütter am Arbeitsplatz, Arbeitszeitregelungen etc. (Skocpol 1992; LaddTaylor 1994). Während der Wirtschaftskrise führten die Frauengruppen, die sich gegen die Ehefrauen diskriminierende Gesetzgebung aussprachen, gezwungenermaßen einen Diskurs über Bedürfnisse: Frauen müßten zur Unterstützung ihrer Familien arbeiten. Das galt selbst für die Frauenpartei und die Anhängerinnen des Equal Rights Amendment. Aber die Gewerkschaften und auch die unorganisierten Arbeiter (besonders alleinstehende Frauen) stellten es so dar, daß erwerbstätige Ehefrauen einen unlauteren Wettbewerb betrieben, um sich Geld für Luxusgüter zu verschaffen. Wenn wir die amerikanische mit der schwedischen Frauenbewegung jener Zeit vergleichen, so zeigt sich, daß die Verwerfungslinien im Zusammenhang mit Arbeitsschutzrechten in Schweden längst keine so große Rolle spielten wie in den USA. Die schwedischen Frauenorganisationen fanden ein gemeinsames Vokabular und einen Themenkreis, der es ihnen ermöglichte, eine breite Koalition von Frauengruppen zu bilden. Daß Organisationen von Frauen aus der Arbeiterklasse bei der Ausgestaltung politischer Identitäten schwedischer Frauen eine Schlüsselrolle innehatten, bedeutete außerdem, daß die politischen Programmen die in Schweden entwickelt wurden, Erfahrungen und Bedürfnisse der Arbeiterinnen wirklich aufgriffen.

\section{Diskursive Felder und die Entstehung von Forderungen}

In der Auseinandersetzung über Bedeutungen findet eine kritische Neufassung der Grenzen von Staatsbürgerrechten statt: Es geht nicht nur darum, wer auf was Anspruch hat, sondern auch um die Grundwerte und politischen Systeme, die die Kriterien dafür liefern, was ein Recht ausmacht, und wodurch das Recht, Rechte zu haben, konstituiert wird. Aus Mangel an stabilen 
institutionellen Grundlagen, mit deren Hilfe sie ihre Ansprüche hätten artikulieren können, waren Frauen in hohem Maße auf diskursive Ressourcen verwiesen, um politische Ziele zu verwirklichen (Fraser 1989). Im Rahmen von Staatsbürgerrechten konnten die schwedischen Feministinnen auf ein traditionelles kulturelles Muster der schwedischen Gesellschaft zurückgreifen: das Volksheim (Folkhem). Sie brachten es auch fertig, einen potentiell konservativen Diskurs über Bevölkerungspolitik zu transformieren und ihn in ihre eigene ideologische Verpackung vom Bürgerrecht auf Arbeit und Familie einzuwickeln: den Grundsatz des Rechts auf Partizipation als Mütter, Arbeiterinnen und Bürgerinnen.

Das schwedische Volksheim war eine Metapher, die in der schwedischen Gesellschaft Anklang fand und die im Laufe der Jahre einen hohen Symbolwert gewonnen hatte. In der öffentlichen Diskussion zuerst von dem konservativen Politiker Rudolph Kjellen verwendet, wurde sie später von den Sozialdemokraten übernommen, als Teil einer Strategie, die eigene Anhängerschaft politisch zu erweitern. Die Metapher vom Volksheim verkörperte einen fürsorglichen Staat, der den Gemeinsinn wiederherstellen würde, und eine Welt, die mit dem Rückgang der Landwirtschaft im Verschwinden begriffen war. Sie sollte Schutz bieten gegenüber all dem, was der schwedischen Gesellschaft in Zeiten rascher Industrialisierung als fremd und allzu wechselhaft erschien (Löfgren 1987). Per Albin Hanssons oft wiederholte Predigt findet ihren Nachhall in den folgenden Bildern:

Die Grundlage des guten Heims ist Gemeinschaft und Solidarität. Im guten Heim gibt es keine Privilegierten oder Benachteiligten, keine Lieblinge und keine Stiefkinder. In einem guten Heim herrschen Gleichheit, Beistand, Kooperation und Hilfsbereitschaft (zit. nach Tingsten 1973, 265).

Den Feministinnen lieferte das Volksheim einen diskursiven Rahmen, innerhalb dessen sie ihre Forderungen nach mehr Teilhaberechten und größerem politischen Einfluß artikulieren konnten. Es versetzte sie in die Lage, die sozialen Alltagserfahrungen mit fürsorgender Arbeit und Kindererziehung mit der politischen Sphäre und der öffentlichen Diskussion zu verbinden. Die Trennung des öffentlichen und des privaten Bereichs des gesellschaftlichen Lebens, die die Grundlage liberaler Theorien von Staatsbürgerschaft bildete, wurde in der Metapher vom Volksheim symbolisch aufgehoben. Und die Frauen fanden einen öffentlichen Raum, um ihre Ansprüche zur Geltung zu bringen, mit denen die Reichweite von Bürgerrechten ausgedehnt wurde auf Fragen von Verhütung und Abtreibung, die Gesundheit von Müttern, Kindertagesstätten und schließlich auch das Recht von erwerbstätigen Frauen, nach Verehelichung und Kindergeburten auf dem Arbeitsmarkt zu bleiben.

Die Feministinnen erkannten, welchen symbolischen Wert das Volksheim hatte, um ihre eigenen Ziele voranzubringen. Ein Artikel in Morgonbris verdeutlicht, wie das Bild des Heims von den Frauen benutzt wurde, um ihre eigene Rolle bei der Ausgestaltung des Wohlfahrtsstaats zu unterstreichen: 
Kaum jemand wird uns jetzt noch dem zentralen Anliegen unserer Agitation öffentlich widersprechen, daß es im eigenen Interesse der Demokratie und der gesellschafilichen Wohlfahrt ist, dem Beitrag von Frauen am kooperativen Aufbau eines sonnigen, gesunden, sicheren und freundlichen Heims für alle ein weiteres Betätigungsfeld zu eröffnen (Morgonbris 1959,1).

Aufgrund des berühmten Buches von Alva und Gunnar Myrdal zur Krise der Bevölkerungsfrage ist die schwedische Bevölkerungsdiskussion ziemlich bekannt. Sie hatten in ihrem Buch vor einem Rückgang der schwedischen Bevölkerung gewarnt und die Auffassung vertreten, der Weg zur Steigerung der Bevölkerungszahl führe über Sozialreformen und die Umverteilung von Ressourcen. Die Beliebtheit ihres Buches und dessen unglaublicher Einfluß auf die schwedische Politik sind nur auf dem Hintergrund zu verstehen, daß sie sich anderen Ideologien so gut anpaßten. Per Albin Hansson, damals schwedischer Ministerpräsident, war einer der ersten, die das Programm der Myrdals aufgriffen. Vielleicht, wie Gunnar Myrdal behauptete, deshalb, weil das "Wohlfahrtsprogramm, das wir voranbringen wollten, auf einer Linie mit Per Albins Traum von einem Heim für das gute Volk lag« (zit. nach Tilton 1991, 147).

Einige der Frauengruppen waren mißtrauisch gegenüber politischen Programmen, die auf Bevölkerungspolitik und Pronatalismus abzielten. Elin Wagner, eine Schülerin Ellen Keys, nannte das einmal "männlichen Dialog " (Lindholm 1992). Alva Myrdal selbst war sich der Gefahren der Bevölkerungsdiskussion sehr wohl bewußt. Die Besorgnis über hohe Arbeitslosigkeit und niedrige Geburtenraten konnte eine gefährliche politische Konstellation einschließen. Aber Alva Myrdal behauptete, daß Frauen in den ideologischen Debatten über Bevölkerung Siegerinnen geworden seien. Im entscheidenden Augenblick sei das Bevölkerungsargument den Antifeministen weggenommen und als neue Waffe für Emanzipationsideale verwendet worden. Der Wandel in der öffentlichen Meinung sei ungeheuerlich gewesen (vgl. Myrdal 1945, 403).

Die meisten aktiven Frauen begriffen, da $\beta$ in der Bevölkerungsfrage ein Potential steckte, mit dem sie Reformen durchbringen konnten, für die sie sich jahrelang eingesetzt hatten. Themen, die früher an Frauenorganisationen oder -kongresse delegiert worden waren, wie Elternurlaub, Kinderversorgung, Verbesserung der Wohnsituation und das Erwerbsrecht von Ehefrauen, wurden nun zu festen Bestandteilen des allgemein-politischen Diskurses. $^{7}$

Zwei Feministinnen bekamen Schlüsselrollen in einem parlamentarischen Ausschuß, der der größeren königlichen Bevölkerungskommission angehörte, die insgesamt achtzehn Berichte veröffentlichte. Vorsitzende des Ausschusses zum Arbeitsrecht von Ehefrauen und Bevölkerung war Kerstin Hesselgren, die erste Frau, die von einer Koalition aus Sozialdemokraten und Liberalen in die erste Kammer des Parlaments gewählt worden war. Sie hatte auch den Vorsitz der linken schwedischen Frauenorganisation, der früheren Freidenkerinnen. Deren Sekretärin Alva Myrdal spielte eine bedeu- 
tende Rolle in der sozialdemokratischen Politik und als Vorsitzende der Vereinigung Berufstätiger Frauen. Abgesehen davon, daß er alle Argumente bezüglich eines unlauteren Wettbewerbs von Frauen um Männerarbeitsplätze entkräftete, befaßte sich der Ausschuß in seinem Bericht (SOU 1938, 47) auch mit Geschlechtsunterschieden im Hinblick auf Arbeitsbedingungen und Löhne. Daß zwei Feministinnen für die Leitung dieses Komitees gewählt wurden, ist ein Zeichen für die Stärke der Frauenorganisationen. Daß sie von den dominierenden politischen Parteien anerkannt wurden, bedeutet auch eine Anerkennung der konstitutiven politischen Kraft der Frauen.

Es lassen sich in dieser Dekade zahlreiche Beispiele finden für den Optimismus, mit dem die Frauen meinten, etwas erreichen zu können. Zum Abschluß der Kommissionsarbeit konnte Myrdal schreiben:

Dies ist ein Jahr sozialpolitischer Pflichten. Wir haben eine Regierung, die auf uns hört. Wir haben ein Parlament, das aufgrund eines angemessenen Sozialprogramms gewählt wurde. Wir haben unter den Frauen politisches Bewußtsein vergrößert und die Familienpolitik steht im Vordergrund (Myrdal, in: Hertha $1937 / 2,1$ ).

Auch ihr Ehemann schlug hohe Töne an und feierte den Erfolg der Frauen, die es geschafft hatten, einen konstitutiven Bestand zu bilden.

Jetzt beginnt die von der Frauenbewegung erfahrene Isolierung aufzubrechen. Frauenfragen sind zu dem geworden, was sie sein sollten, ein wichtiger, nicht abtrennbarer Bestandteil der Gesamtgesellschaft. Mit diesen Mitteln kann die Frauenbewegung nur gewinnen (Hertha 1935/4, 82).

\section{Politische Chancen}

Die Dreißiger waren für die schwedischen Feministinnen eine außergewöhnliche Zeit: es war eine Periode des durchlässigen und offenen politischen Raums, in dem wieder eine vitale Form weiblicher Staatsbürgerschaft auftauchen konnte, etwas, das Hannah Arendt einen verlorenen Schatz der Demokratie nennen würde. Es war auch eine Zeit, in der sich Wohlfahrtsstaaten entwickeln und ausweiten konnten, es gab Platz für soziale Gruppen, neue Ansprüche geltend $\mathrm{zu}$ machen und die Bürgerrechte zu erweitern. Schließlich war es auch eine Periode in der schwedischen Politik, in der politische Parteien keine Mehrheiten hinter sich hatten, und es war auch noch keineswegs sicher, daß die Sozialdemokraten sich so lange halten würden. Das bekannte Saltsjöbaden-Abkommen, mit dem eine korporative Machtstruktur zwischen Unternehmen, Gewerkschaften und Regierung zustande kam, wurde erst 1938 unterzeichnet.

Sidney Tarrow (1994) bemerkt sehr scharfsinnig, daß es eine nicht-lineare Beziehung zwischen der Organisation sozialer Bewegungen und der Aufgeschlossenheit des Staates gibt. Zahlreiche historische Untersuchungen zu 
Frauenbewegungen zeigen in aller Deutlichkeit: Mobilisierung kann durch Mangel an Verbündeten in der Legislative paralysiert oder durch konkurrierende Diskurse anderer oppositioneller Gruppen zerschlagen werden. Hier kommen nun die politischen »Gelegenheitsstrukturen « ins Spiel: In Schweden hatte sich die politische Umgebung sehr günstig entwickelt für eine Mobilisierung von Frauen, die in politische Handlungsfähigkeit einmündete. Aber abgesehen von dem Vermögen sozialer Gruppen, aus günstigen politischen Umständen Kapital zu schlagen, gibt es das noch provokativere Theorem von einer Kausalbeziehung zwischen politischen Chancen und der $\mathrm{Zu}$ sammensetzung konstitutiv handelnder Gruppen. Der Fall der Mobilisierung schwedischer Frauen, mit dem wir es hier zu tun haben, läßt darauf schließen, daß die kognitive Verfassung einer Bewegung und die verwendeten Diskursstrategien politische Gelegenheiten erschließen können. Politische Gelegenheiten lassen sich tatsächlich nicht trennen von der Herstellung politischer Identitäten und dem Aufbau politisch handlungsfähiger $\mathrm{Be}$ wegungen. Dies ist der Kernbereich gegenwärtiger Forschung, wenn es um die Kopplung partizipatorischer und sozialer Bürgerrechte geht.

\section{$\operatorname{Schlu} \beta$}

Während der dreißiger Jahre konnten die schwedischen Frauen mit großem Erfolg politische Strategien durchsetzen, die zum Kern weiblicher Staatsbürgerrechte im schwedischen Wohlfahrtsstaat werden sollten: Mutterschaftsurlaub und Arbeitsplatzsicherheit, gesetzlicher Schutz des Rechts auf Erwerbstätigkeit von Ehefrauen, Einkommensgarantien für ledige Mütter und allgemeine Gesundheitsfürsorge für Mütter. Bedarfsorientierte Beihilfen für Mütter wurden ebenfalls gesetzlich geregelt. Tatsächlich bekam die Mehrheit aller Mütter diese Zuschüsse (Ohlander 1992). Wenn auch Arbeitslosigkeit und Renten bei den Gesamtkosten faktisch höhere Ausgaben erforderten, so stellten doch die gesetzlichen Maßnahmen und ein Großteil der Diskussionen über Bürgerrechte von Frauen eine Ausweitung des Sozialstaats dar. Dieser bewegte sich in neuen Terrains und vergrößerte die sozialen Rechte von Frauen: die Beihilfen wurden direkt an die Frauen verteilt und waren so ausgestaltet, daß sie autonome Haushalte gründen konnten (Hobson 1990; Orloff 1993). Diese politischen Initiativen und Debatten legten den Grund für eine Reihe weiterer Initiativen, aus denen sehr großzügige Elternurlaubsregelungen hervorgingen. Im Anschluß entstanden staatlich unterstützte Kindertagesstätten und ein Altenpflegesystem, das Arbeitsplätze für Frauen im öffentlichen Sektor schuf. Die Konturen weiblicher Staatsbürgerschaft können bis in die Periode zurückverfolgt werden, in der das Bürgerrecht auf Partizipation einen Bedeutungswandel erfuhr, es hieß jetzt: in den Sphären der Politik, der bezahlten Arbeit und der Familienarbeit gleichermaßen zu partizipieren. Die bürgerrechtlichen Aspekte, die sich damals herauskristallisierten, werden sichtbar an den Folgen des schwedischen Modells von zwei Familienernährern: Die große Zahl stellensuchen- 
der Mütter mit Kleinkindern auf dem schwedischen Arbeitsmarkt und die Vielzahl teilzeitarbeitender Mütter sind Indizien dafür, daß das Ziel sozialer und ökonomischer Gleichheit zwischen Männern und Frauen, das seinerzeit im "Aufruf an die schwedischen Frauen « formuliert worden war, nicht erreicht wurde. Eine andere Hinterlassenschaft dieser Zeit, unter allen westlichen Demokratien in Schweden die höchste Zahl von Frauen im Parlament zu haben, macht deutlich, welche Relevanz der Bezugsrahmen Bürgerrecht als mobilisierende Kraft in der schwedischen Politik hatte. In ihrer positiven Beurteilung des skandinavischen Wohlfahrtsstaats hat Helga Hernes (1987) bemerkt, daß Frauen infolge der Ausweitung ihrer Bürgerrechte mobilisiert worden seien, anders gesagt, durch Interaktion mit öffentlicher Politik. Wenn dies auch in mancher Hinsicht zutreffend sein mag, so zeigt die hier vorgelegte Analyse, daß der Grund für politische Teilhabe- und soziale Bürgerrechte früher gelegt wurde und daß die frauenfreundliche Sozialpolitik das Ergebnis von früheren Erfolgen der Frauen ist, politische Handlungsfähigkeit zu entwickeln.

\section{Aus dem Amerikanischen übersetzt von Regine Othmer.}

\section{Anmerkungen}

1 Es gibt eine umfangreiche Literatur zu Geschlechterverhältnissen und Staatsbürgerschaft: vgl. Pateman mit ihrer bahnbrechenden Arbeit über klassische Texte (1988); zur Analyse geschlechtsneutraler Konzepte bei Marshall vgl. Fraser und Gordon (1994), Hobson (1994), Orloff (1993) und Pedersen (1994); Mueller Okin (1989) hat eine sehr aufschlußreiche Kritik an Rawls formuliert.

2 Die Behandlung des schwedischen Beispiels stützt sich auf Untersuchungen von Hobson und Lindholm, im Erscheinen (1996).

3 Norwegen macht hier eine Ausnahme, da dort tatsächlich maternalistische Themen grundlegend für den Feminismus waren (vgl. Leira 1992).

4 Ellen Key kann als letzte Maternalistin angesehen werden. Sie war mehr als kritisch gegenüber allen feministischen Versuchen, Einfluß auf die männlichen Domänen der Politik und des Arbeitsmarkts zu gewinnen (vgl. Key 1912).

5 Interessant ist, daß die Angriffe auf das Recht der Frauen auf Erwerbsarbeit in verschiedenen Ländern keineswegs mit dem Niveau der Arbeitslosigkeit zusammenhängen oder mit der Zahl arbeitender Ehefrauen (Hobson 1993).

6 Es war bei einigen Fabriken geläufige Praxis, Frauen hinauszuwerfen, wenn sie heirateten. Versicherungsgesellschaften und Banken hatten es zur Regel gemacht, Frauen fünf Monate nach der Heirat zu entlassen (Wikander 1991,39).

7 Die Frauengruppen mußten ihre Forderung nach einem liberaleren Abtreibungsgesetz im Zuge der hitzigen Debatte über Bevölkerungsrückgang aufgeben, aber sie konnten durchsetzen, daB Informationen zur Verhütung verbreitet wurden. 


\section{Literatur}

Arbetets Kvinnor (1932): Utredning om Gift Kvinnas Statsjänst. March: 4-5.

De Swann (1995): The Receding Prospects for Transnational Social Policy. Paper presented at the Social Science History Conference, November 10-13, Chicago Illiniois.

Dietz, Mary (1992): Context is All: Feminism and Theories of Citizenship. In: Dimensions of Radical Democracy: Pluralism, Citizenship and Community. Edited by Chantal Mouffe. London: Verso.

Eskillsson, Lena (1991): Drömmen om Kamratsamhället. Carlssons.

Flood, Hulda (1960): Den socialdemokratiska kvinnorörelsen in Sverige. Stockholm: Tiden.

Evans, Richard J. (1977): The Feminists: Women's Emancipation Movements in Europe, America and Australia 1840-1920. London.

Fraser, Nancy (1989): Unruly Practices, Power Discourse and Gender in Contemporary Social Theory. Minneapolis: University of Minnesota Press.

Fraser, Nancy and Linda Gordon (1994): „Dependency « Demystified: Inscriptions of Power in a Keyword of the Welfare State. Social Politics: International Studies in Gender, State, and Society. $1: 1432$.

Harris, Cynthia (1988): On Account of Sex. The Politics of Women's Issues. Berkeley, California: University Press.

Hernes, Helga (1987): Welfare State and Woman Power: Essays in State Feminism. Oslo.

Hobson, Barbara (1993): "Feminist Strategies and Gendered Discourses in Welfare States: Married Women's Right to Work in the U.S. and Sweden During the 1930's. « pp. 396-429. In Mothers of a New World. Maternalist Politics and the Origins of Welfare States. Edited by Seth Koven and Sonya Michel. NY: Routledge, Kegan and Paul.

Hobson, Barbara (1990): No Exit No Voice: women's economic dependency and the welfare state. Acta Sociologica. 33:3: 235-250.

Hobson, Barbara and Marika Lindholm (1996): Forthcoming. »Women's Power Resources and the Construction of Citizenship Rights in Welfare States. "Theory and Society.

Honig, Bonnie (ed.) (1995): Feminist Interpretations of Hannah Arendt. University Park, Pa.: Pennsylvania State University.

Jones, Jaqueline and Kahleen B. Jones (1994): Identity, Action, and Locale. Social Politics: International Studies in Gender, State, and Society. 1:3:256-271.

Key, Ellen (1912): The Woman Movement. New York: Putnam.

Knijn, Trudie (1994): Fish Without Bikes: Revision of the Dutch Welfare State and its Consequences for the (In)dependence of Single Mothers. Social Politics. 1:1 83-105.

Kock, Karin (1935): »Kris i Befolkningsfrågan.« Husmodersförbundets Medlemsblad. February: $1,4 \& 5$.

Koven, Seth and Sonya Michel (eds) (1993): Mothers of a New World: Maternalist Politics. In: Mothers of a New World. Maternalist Politics and the Origins of Welfare States. NY: Routledge, Kegan and Paul.

Ladd-Taylor, Molly (1994): Mother-Work: Women, Child Welfare, and the State, 1890-1930. Urbana: University of Illinois Press.

Landes, Joan B. (1995): Novus Ordo Saeclorum: Gender and Public Space in Arendt's Revolutionary France. In: Feminist Interpretations of Hanna Arendt. Edited by Bonnie Honnig. University Park, Pa.: Pennsylvania State University.

Lindholm, Margaretha (1990): Talet Om Det Kvinnliga. University of Gothenberg.

Lindholm, Margaretha (1992): Elin Wägner och Alva Myrdal. Uddevalla: Anamma förlag.

Lindholm, Marika (1991): „Swedish Feminism, 1835-1945: A Conservative Revolution.» Journal of Historical Sociology. 4, 2:121-142.

Lister, Ruth (1996): Dilemmas in Engendering Citizenship. Economy and Society. 24: 1, 1-40.

Lösman, Beata (1987): Kvinnoorganisering och Kvinnorörelser i Sverige. In: Gunhild Kyle (ed.): Handbok i Svensk Kvinnohistoria. Stockholm: Carlssons.

Löfgren, Orvar and Jonas Frykman (1987): Culture Builders: A Historical Anthropology of Middle-Class Life. trans. Alan Crozier. New Brunswick: Rutgers University Press. 
Marshall, T. H. (1950): Citizenship and Social Class and Other Essays. Cambridge, England: Cambridge University Press.

Morgonbris (1935): „Folkets Framtid. « January: 5-8, 1939. "Kvinnoarbetskommittens betänkande - intressant och spännande läsning. « February: 4-6 \& 26: 1939. "Kvinnorörelsen.“ December: $5,20 \& 25$.

Myrdal, Alva (1945): Nation and Family. London: Kegan Paul, Trench, Trubner, \& Co.

Myrdal, Gunnar (1935): „Befolkningsfrägan och Kvinnofrägan.« Hertha. April: 81-82.

Ohlander, Ann-Sofie (1992): The Invisible Child: The Struggle Over Social Democratic Family Policy.« pp. 213-236. In: Creating Social Democracy: A Century of the Social Democratic Party in Sweden. Edited by Klaus Misgeld, Karl Molin, and Klas Amark. University Park, Pa: Penn State Press.

Orloff, Ann Shola (1993): Gender and the Social Rights of Citizenship: The Comparative Analysis of State Policies and Gender Relations. American Sociological Review. 5: 303-328.

Phillips, Anne (1991): Engendering Democracy. Cambridge, England: Basic Blackwell.

Rawls, John (1971): $A$ Theory of Justice: Cambridge: Harvard University Press.

Saraceno, Chiara (1994): The Ambivalent Famialism of the Italian Welfare State. Social Politics. International Studie of Gender, State and Society. 1:1:60-82.

Siim, Birte (1994): Engendering Democracy: Social Citizenship and Political Participation for Women in Scandinavia. Social Politics. International Studies in Gender, State, and Society. 1:3 286-305.

Skocpol, Theda (1992): Protecting Soldiers and Mothers. Cambridge, MA: Harvard University Press.

Snow, David A. and Robert D. Benford (1992): Master Frames and Cycles of Protest. pp. 133-155. In: Frontiers in Social Movement Theory. Edited by A. D. Morris and C. M. Mueller. New Haven: Yale University Press.

Snow, David A., E. Burke Rochford, Jr., Steven K. Worden, and Robert D. Benford (1986): Frame Allignment and Mobilization. American Sociological Review 51, 4:464-481.

SOU (1938): 13. Betankande angaende forvarsvsarbetande kvinnors rattsliga stallning vid atktenskap or barnsbord.

Spoof, C. (1933): „Kvinnorna Behöver sina Verkstäder«. Tidevarvet. July: 1.

Svenska Kvinnors Medborgarsförbund. 1931: 23.

Tarrow, Sydney (1994): Power in Movement: Social Movements, Collective Action, and Politics. Cambridge, England: Cambridge University Press.

Thorell, Ruth Hamrin (1969): "Ruth Hamrin Thorell i Riksdagen. Hertha. February: 23-24.

Tilton, Tim (1991): The Political Theory of Swedish Social Democracy. Oxford: Clarendon Press.

Tingsten, Herbert (1973): The Swedish Social Democrats: Their Ideological Development. Trans. Greta Frankel and Patricia Howard-Rosen. New Jersey: Bedminster Press.

Turner, Bryan (1992): Outline of a Theory of Citizenship. In: Dimensions of Radical Democracy: Pluralism, Citizenship, and Community. Edited by Chantal Mouffe. London: Verso.

Wikander, Ulla (1992): „Kvinnorna $\mathrm{i}$ den Tidiga Industrialiseringen.« In: Kvinnohistoria. Stockholm: Utbildningsradion.

Wohlin, Margit (1935): Hemarbetande och Förvärvsarbetande. Husmodersförbundets Medlemsblad. May: 6-7.

Åkerman, Brita (1983): Vi Kan, Vi Behovs! - Kvinnorna går samman i egna föreningar. Stockholm: Förlaget Akademilitteratur. 